\title{
Semirelativistic 1s-2s Excitation of Atomic Hydrogen by Electron Impact
}

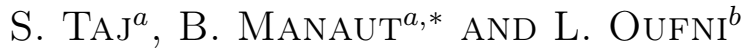 \\ ${ }^{a}$ Faculté Polydisciplinaire, Université Sultan Moulay Slimane, Laboratoire Interdisciplinaire de Recherche \\ en Sciences et Techniques (LIRST), Boite Postale 523, 23000 Béni Mellal, Morocco \\ ${ }^{b}$ Faculté des Sciences et Techniques, Département de Physique, Université Sultan Moulay Slimane, \\ LPMM-ERM, Boite Postale 523, 23000 Béni Mellal, Morocco
}

(Received September 21, 2010; in final form December 15, 2010)

\begin{abstract}
In the framework of the first Born approximation, we present a semirelativistic theoretical study of the inelastic excitation $\left(1 s_{1 / 2} \longrightarrow 2 s_{1 / 2}\right)$ of hydrogen atom by electronic impact. The incident and scattered electrons are described by a free Dirac spinor and the hydrogen atom target is described by the Darwin wave function. Relativistic and spin effects are examined in the relativistic regime. A detailed study has been devoted to the nonrelativistic regime as well as the moderate relativistic regime. Some aspects of this dependence as well as the dynamic behavior of the differential cross-section in the relativistic regime have been addressed.
\end{abstract}

PACS: $34.80 . D p, 12.20 . D s$

\section{Introduction}

The theoretical study of relativistic electron-atom collisions is fundamental to our understanding of many aspects in plasma physics and astrophysics. The development of electron-atom collision studies has also been strongly motivated by the need of data for testing and developing suitable theories of the scattering and collision process, and providing a tool for obtaining detailed information on the structure of the target atoms and molecules. Many authors have studied this process using numerical tools. Thus, Kisielius et al. [1] employed the $R$-matrix method with nonrelativistic and relativistic approximations for the hydrogen-like $\mathrm{He}^{+}, \mathrm{Fe}^{25+}$ and $\mathrm{U}^{91+}$ ions, where the case of transitions $1 s \longrightarrow 2 s$ and $1 s \longrightarrow 2 p$ as well as those between fine structure $n=2$ levels were considered. Andersen and Bartschat [2] have applied the semirelativistic Breit-Pauli $R$-matrix to calculate the electron-impact excitation of the ${ }^{2} S_{1 / 2} \longrightarrow$ ${ }^{2} P_{1 / 2,3 / 2}^{\mathrm{o}}$ resonance transitions in heavy alkali atoms. Payne et al. [3] have studied the electron-impact excitation of the $5 s \longrightarrow 5 p$ resonance transition in rubidium by using a semirelativistic Breit-Pauli $R$-matrix with pseudo-states (close-coupling) approach. Attaourti et al. [4] have investigated the exact analytical relativistic excitation $1 S_{1 / 2} \longrightarrow 1 S_{1 / 2}$ of atomic hydrogen by electron impact in the presence of a laser field. They have found that a simple formal analogy links the analytical expressions of the unpolarized differential cross-section (DCS) without laser and the unpolarized differential cross section in the presence of a laser field.

* corresponding author; e-mail: manaut@fstbm.ac.ma
The aim of this contribution is to add some new physical insights and to show that the nonrelativistic formalism enables to describe particles with high kinetic energies. Before we present the results of our investigation, we first begin by sketching the main steps of our treatment. For pedagogical purposes, we begin by the most basic results of our work using atomic units (a.u.) in which one has $\hbar=m_{\mathrm{e}}=e=1$, where $m_{\mathrm{e}}$ is the electron mass at rest, and which will be used throughout this work. We will also work with the metric tensor $g^{\mu \nu}=\operatorname{diag}(1,-1,-1,-1)$ and the Lorentz scalar product which is defined by $(a \cdot b)=a^{\mu} b_{\mu}$. The layout of this paper is as follows. We present the necessary formalism of this work in Sects. 2, 3 and 4, the result and discussion in Sect. 5 and we end by a brief conclusion in Sect. 6 .

\section{Theory of the inelastic collision $1 s_{1 / 2} \longrightarrow 2 s_{1 / 2}$}

In this section, we calculate the exact analytical expression of the semirelativistic unpolarized DCS for the relativistic excitation of atomic hydrogen by electron impact. The transition matrix element for the direct channel (exchange effects are neglected) is given by

$$
\begin{aligned}
S_{\mathrm{fi}} & =-\mathrm{i} \int \mathrm{d} t\left\langle\psi_{p_{\mathrm{f}}}\left(x_{1}\right) \phi_{\mathrm{f}}\left(x_{2}\right)\left|V_{\mathrm{d}}\right| \psi_{p_{\mathrm{i}}}\left(x_{1}\right) \phi_{\mathrm{i}}\left(x_{2}\right)\right\rangle \\
& =-\mathrm{i} \int_{-\infty}^{+\infty} \mathrm{d} t \int \mathrm{d} \boldsymbol{r}_{1} \bar{\psi}_{p_{\mathrm{f}}}\left(t, \boldsymbol{r}_{1}\right) \gamma^{0} \psi_{p_{\mathrm{i}}}\left(t, \boldsymbol{r}_{1}\right) \\
& \times\left\langle\phi_{\mathrm{f}}\left(x_{2}\right)\left|V_{\mathrm{d}}\right| \phi_{\mathrm{i}}\left(x_{2}\right)\right\rangle,
\end{aligned}
$$

where 


$$
V_{\mathrm{d}}=\frac{1}{r_{12}}-\frac{Z}{r_{1}}
$$

is the direct interaction potential, $\boldsymbol{r}_{1}$ are the coordinates of the incident and scattered electron, $\boldsymbol{r}_{2}$ the atomic electron coordinates, $r_{12}=\left|\boldsymbol{r}_{1}-\boldsymbol{r}_{2}\right|$ and $r_{1}=\left|\boldsymbol{r}_{1}\right|$. The function $\psi_{p_{\mathrm{i}}}\left(x_{1}\right)=\psi_{p}\left(t, \boldsymbol{r}_{1}\right)=u(p, s) \exp (-\mathrm{i} p \cdot x) / \sqrt{2 E V}$ is the electron wave function, described by a free Dirac spinor normalized to the volume $V$, and $\phi_{\mathrm{i}, \mathrm{f}}\left(x_{2}\right)=$ $\phi_{\mathrm{i}, \mathrm{f}}\left(t, \boldsymbol{r}_{2}\right)$ are the semirelativistic wave functions of the hydrogen atom where the index $\mathrm{i}$ and $\mathrm{f}$ stand for the initial and final states, respectively. The semirelativistic wave function of the atomic hydrogen is the Darwin wave function for bound states [5], which is given by

$$
\phi_{\mathrm{i}}\left(t, \boldsymbol{r}_{2}\right)=\exp \left(-\mathrm{i} \mathcal{E}_{\mathrm{b}}\left(1 s_{1 / 2}\right) t\right) \varphi_{1 s}^{( \pm)}\left(\boldsymbol{r}_{2}\right),
$$

where $\mathcal{E}_{\mathrm{b}}\left(1 s_{1 / 2}\right)$ is the binding energy of the ground state of atomic hydrogen and $\varphi_{1 s}^{( \pm)}\left(\boldsymbol{r}_{2}\right)$ is given by

$$
\varphi_{1 s}^{( \pm)}\left(\boldsymbol{r}_{2}\right)=\left(1_{4}-\frac{\mathrm{i}}{2 c} \alpha \cdot \nabla_{(2)}\right) u^{( \pm)} \varphi_{0}\left(\boldsymbol{r}_{2}\right),
$$

it represents a quasirelativistic bound state wave function, accurate to first order in $Z / c$ in the relativistic corrections (and normalized to the same order), with $\varphi_{0}$ being the nonrelativistic bound state hydrogenic function. The spinors $u^{( \pm)}$are such that $u^{(+)}=(1,0,0,0)^{\mathrm{T}}$ and $u^{(-)}=(0,1,0,0)^{\mathrm{T}}$ and represent the basic four-component spinors for a particle at rest with spin-up and spin-down, respectively. The matrix differential operator $\alpha \cdot \nabla$ is given by

$$
\alpha \cdot \nabla=\left(\begin{array}{cccc}
0 & 0 & \partial_{z} & \partial_{x}-\mathrm{i} \partial_{y} \\
0 & 0 & \partial_{x}+\mathrm{i} \partial_{y} & -\partial_{z} \\
\partial_{z} & \partial_{x}-\mathrm{i} \partial_{y} & 0 & 0 \\
\partial_{x}+\mathrm{i} \partial_{y} & -\partial_{z} & 0 & 0
\end{array}\right) .
$$

For the spin up, we have

$$
\varphi_{1 s}^{(+)}\left(\boldsymbol{r}_{2}\right)=N_{\mathrm{D}_{1}}\left(\begin{array}{c}
1 \\
0 \\
\frac{\mathrm{i}}{2 c r_{2}} z \\
\frac{\mathrm{i}}{2 c r_{2}}(x+\mathrm{i} y)
\end{array}\right) \frac{1}{\sqrt{\pi}} \mathrm{e}^{-r_{2}},
$$

and for the spin down, we have

$$
\varphi_{1 s}^{(-)}\left(\boldsymbol{r}_{2}\right)=N_{\mathrm{D}_{1}}\left(\begin{array}{c}
0 \\
1 \\
\frac{\mathrm{i}}{2 c r_{2}}(x-\mathrm{i} y) \\
-\frac{\mathrm{i}}{2 c r_{2}} z
\end{array}\right) \frac{1}{\sqrt{\pi}} \mathrm{e}^{-r_{2}},
$$

where

$$
N_{\mathrm{D}_{1}}=2 c / \sqrt{4 c^{2}+1}
$$

is a normalization constant lower but very close to 1 . Let us mention that the function $\phi_{\mathrm{f}}\left(t, \boldsymbol{r}_{2}\right)$ in Eq. (1) is the Darwin wave function for bound states [6], which is also accurate to the order $Z / c$ in the relativistic corrections. This is expressed as $\phi_{\mathrm{f}}\left(t, \boldsymbol{r}_{2}\right)=$ $\exp \left(-\mathrm{i} \mathcal{E}_{\mathrm{b}}\left(2 s_{1 / 2}\right) t\right) \varphi_{2 s}^{( \pm)}\left(\boldsymbol{r}_{2}\right)$ with $\mathcal{E}_{\mathrm{b}}\left(2 s_{1 / 2}\right)$ as the binding energy of the $2 s_{1 / 2}$ state of atomic hydrogen

$$
\varphi_{2 s}^{(+)}\left(\boldsymbol{r}_{2}\right)=N_{\mathrm{D}_{2}}\left(\begin{array}{c}
2-r_{2} \\
0 \\
\mathrm{i} \frac{4-r_{2}}{4 r_{2} c} z \\
\frac{4-r_{2}}{4 r c}(-y+\mathrm{i} x)
\end{array}\right) \frac{1}{4 \sqrt{2 \pi}} \mathrm{e}^{-r_{2}}
$$

for the spin up and

$$
\varphi_{2 s}^{(-)}\left(\boldsymbol{r}_{2}\right)=N_{\mathrm{D}_{2}}\left(\begin{array}{c}
0 \\
2-r_{2} \\
\frac{4-r_{2}}{4 c r_{2}}(y+\mathrm{i} x) \\
\mathrm{i} \frac{r_{2}-4}{4 c r_{2}} z
\end{array}\right) \frac{1}{4 \sqrt{2 \pi}} \mathrm{e}^{-r_{2}}
$$

for the spin down. The transition matrix element in Eq. (1) becomes

$$
\begin{aligned}
& S_{\mathrm{fi}}=-\mathrm{i} \int_{-\infty}^{+\infty} \mathrm{d} t \int \mathrm{d} \boldsymbol{r}_{1} \mathrm{~d} \boldsymbol{r}_{2} \bar{\psi}_{p_{\mathrm{f}}}\left(t, \boldsymbol{r}_{1}\right) \gamma^{0} \psi_{p_{\mathrm{i}}}\left(t, \boldsymbol{r}_{1}\right) \\
& \quad \times \phi_{\mathrm{f}}^{\dagger}\left(t, r_{2}\right) \phi_{\mathrm{i}}\left(t, r_{2}\right) V_{\mathrm{d}}
\end{aligned}
$$

and it is straightforward to get, for the transition amplitude,

$$
\begin{aligned}
& S_{\mathrm{fi}}=-\mathrm{i} \frac{\bar{u}\left(p_{\mathrm{f}}, s_{\mathrm{f}}\right) \gamma^{0} u\left(p_{\mathrm{i}}, s_{\mathrm{i}}\right)}{2 V \sqrt{E_{\mathrm{f}} E_{\mathrm{i}}}} 2 \pi H_{\text {inel }}(\Delta) \\
& \quad \times \delta\left(E_{\mathrm{f}}+\mathcal{E}\left(2 s_{1 / 2}\right)-E_{\mathrm{i}}-\mathcal{E}\left(1 s_{1 / 2}\right)\right),
\end{aligned}
$$

where $\Delta=\left|p_{\mathrm{i}}-p_{\mathrm{f}}\right|$ and $\gamma^{0}$ is the Dirac matrix. Using the standard technique of the QED, we find for the unpolarized DCS

$$
\begin{aligned}
& \frac{\mathrm{d} \bar{\sigma}}{\mathrm{d} \Omega_{\mathrm{f}}}=\frac{\left|\boldsymbol{p}_{\mathrm{f}}\right|}{\left|\boldsymbol{p}_{\mathrm{i}}\right|} \frac{1}{\left(4 \pi c^{2}\right)^{2}}\left(\frac{1}{2} \sum_{s_{\mathrm{i}} s_{\mathrm{f}}}\left|\bar{u}\left(p_{\mathrm{f}}, s_{\mathrm{f}}\right) \gamma^{0} u\left(p_{\mathrm{i}}, s_{\mathrm{i}}\right)\right|^{2}\right) \\
& \quad \times\left|H_{\text {inel }}(\Delta)\right|^{2} .
\end{aligned}
$$

\section{Calculation of the integral part}

The function $H_{\text {inel }}(\Delta)$ is found if one performs the various integrals

$$
H_{\text {inel }}(\Delta)=\int_{0}^{+\infty} \mathrm{d} \boldsymbol{r}_{1} \mathrm{e}^{\mathrm{i} \Delta \boldsymbol{r}_{1}} I\left(\boldsymbol{r}_{1}\right) .
$$

\subsection{Integral over $\boldsymbol{r}_{2}$}

The quantity $I\left(\boldsymbol{r}_{1}\right)$ is easily evaluated in the following way. We first write the explicit form of $I\left(\boldsymbol{r}_{1}\right)$ :

$$
I\left(\boldsymbol{r}_{1}\right)=\int_{0}^{+\infty} \mathrm{d} \boldsymbol{r}_{2} \phi_{2 s}^{\dagger}\left(\boldsymbol{r}_{2}\right)\left(\frac{1}{r_{12}}-\frac{Z}{r_{1}}\right) \phi_{1 s}\left(\boldsymbol{r}_{2}\right) .
$$

Next, we develop the quantity $r_{12}^{-1}$ in spherical harmonics as

$$
\frac{1}{\boldsymbol{r}_{12}}=4 \pi \sum_{l m} \frac{Y_{l m}\left(\widehat{r}_{1}\right) Y_{l m}^{*}\left(\widehat{r}_{2}\right)}{2 l+1} \frac{\left(\boldsymbol{r}_{<}\right)^{l}}{\left(\boldsymbol{r}_{>}\right)^{l+1}},
$$

where $r_{>}$is the greater of $r_{1}$ and $r_{2}$, and $r_{<}$the lesser of 
them. The angular coordinates of the vectors $\boldsymbol{r}_{1}$ and $\boldsymbol{r}_{2}$ are such that: $\widehat{r}_{1}=\left(\theta_{1}, \varphi_{1}\right)$ and $\widehat{r}_{2}=\left(\theta_{2}, \varphi_{2}\right)$. We use the well known integral [7]:

$$
\begin{aligned}
& \int_{x}^{+\infty} \mathrm{d} u u^{m} \mathrm{e}^{-\alpha u}=\frac{m !}{\alpha^{m+1}} \mathrm{e}^{-\alpha x} \sum_{\mu=0}^{m} \frac{\alpha^{\mu} x^{\mu}}{\mu !}, \\
& \operatorname{Re}(\alpha)>0,
\end{aligned}
$$

then, after some analytic calculations, we get for $I\left(\boldsymbol{r}_{1}\right)$ :

$$
I\left(\boldsymbol{r}_{1}\right)=\frac{6}{27}\left(\frac{1}{c^{2}}-4\right)+\frac{4}{27 c^{2}} \frac{1}{\boldsymbol{r}_{1}}-\frac{4}{9}\left(1+\frac{1}{8 c^{2}}\right) \boldsymbol{r}_{1} .
$$

\subsection{Integral over $\boldsymbol{r}_{1}$}

The integration over $\boldsymbol{r}_{1}$ gives rise to the following formula:

$$
\begin{aligned}
& H_{\text {inel }}(\Delta)=\int_{0}^{+\infty} \mathrm{d} \boldsymbol{r}_{1} \mathrm{e}^{\mathrm{i} \Delta \boldsymbol{r}_{1}} I\left(\boldsymbol{r}_{1}\right) \\
& =-\frac{4 \pi}{\sqrt{2}}\left(I_{1}+I_{2}+I_{3}\right)
\end{aligned}
$$

the angular integrals are performed by expanding the plane wave $\mathrm{e}^{\mathrm{i} \boldsymbol{\Delta} \boldsymbol{r}_{1}}$ in spherical harmonics as

$$
\mathrm{e}^{\mathrm{i} \boldsymbol{\Delta} \boldsymbol{r}_{1}}=\sum_{l m} 4 \pi \mathrm{i}^{l} j_{l}\left(\boldsymbol{\Delta} \boldsymbol{r}_{1}\right) Y_{l m}(\widehat{\boldsymbol{\Delta}}) Y_{l m}^{*}\left(\widehat{\boldsymbol{r}}_{1}\right)
$$

with $\boldsymbol{\Delta}=\boldsymbol{p}_{\mathrm{i}}-\boldsymbol{p}_{\mathrm{f}}$ is the relativistic momentum transfer and $\widehat{\Delta}$ is the angular coordinate of the vector $\boldsymbol{\Delta}$. Then, after some analytic computations, we get for $I_{1}, I_{2}$ and $I_{3}$ the following result:

$$
\begin{aligned}
I_{1} & =\frac{4}{27 c^{2}} \int_{0}^{+\infty} \mathrm{d} r_{1} r_{1} \mathrm{e}^{-\frac{3}{2} r_{1}} j_{0}\left(\Delta r_{1}\right) \\
& =\frac{4}{27 c^{2}} \frac{1}{\left[(3 / 2)^{2}+\Delta^{2}\right]}, \\
I_{2} & =\frac{6}{27}\left(\frac{1}{c^{2}}-4\right) \int_{0}^{+\infty} \mathrm{d} r_{1} r_{1}^{2} \mathrm{e}^{-\frac{3}{2} r_{1}} j_{0}\left(\Delta r_{1}\right) \\
& =\frac{2}{27}\left(\frac{1}{c^{2}}-4\right) \frac{3}{\left[(3 / 2)^{2}+\Delta^{2}\right]}, \\
I_{3} & =-\frac{4}{9}\left(1+\frac{1}{8 c^{2}}\right) \int_{0}^{+\infty} \mathrm{d} r_{1} r_{1}^{3} \mathrm{e}^{-\frac{3}{2} r_{1}} j_{0}\left(\Delta r_{1}\right) \\
& =\frac{8}{9}\left(1+\frac{1}{8 c^{2}}\right) \frac{\Delta^{2}-27 / 4}{\left[(3 / 2)^{2}+\Delta^{2}\right]} .
\end{aligned}
$$

It is clear that the situation is different than in elastic collision [4], since we have no singularity in the case $\boldsymbol{\Delta} \rightarrow 0$.

\section{Calculation of the spinorial part}

The calculation is now reduced to the computation of traces of $\gamma$ matrices. This is routinely done using Reduce [8]. We consider the unpolarized DCS. Therefore, the various polarization states have the same probability and the actual calculated spinorial part is given by summing over the final polarization $s_{\mathrm{f}}$ and averaging aver the initial polarization $s_{\mathrm{i}}$. Therefore, the spinorial part is given by

$$
\begin{aligned}
& \frac{1}{2} \sum_{s_{\mathrm{i}} s_{\mathrm{f}}}\left|\bar{u}\left(p_{\mathrm{f}}, s_{\mathrm{f}}\right) \gamma^{0} u\left(p_{\mathrm{i}}, s_{\mathrm{i}}\right)\right|^{2} \\
& \quad=\operatorname{Tr}\left(\gamma^{0}\left(\not p_{\mathrm{i}} c+c^{2}\right) \gamma^{0}\left(\not p_{\mathrm{f}} c+c^{2}\right)\right) \\
& \quad=2 c^{2}\left(\frac{2 E_{\mathrm{f}} E_{\mathrm{i}}}{c^{2}}-\left(p_{\mathrm{i}} \cdot p_{\mathrm{f}}\right)+c^{2}\right) .
\end{aligned}
$$

We must, of course, recover the result in the nonrelativistic limit $(\gamma \longrightarrow 1)$, situation of which the differential cross-section can be simply given by

$$
\frac{\mathrm{d} \bar{\sigma}}{\mathrm{d} \Omega_{\mathrm{f}}}=\frac{\left|\boldsymbol{K}_{\mathrm{f}}\right|}{\left|\boldsymbol{K}_{\mathrm{i}}\right|} \frac{128}{\left(\left|\boldsymbol{\Delta}_{\mathrm{nr}}\right|^{2}+\frac{9}{4}\right)^{6}}
$$

with $\left|\boldsymbol{\Delta}_{\mathrm{nr}}\right|=\left|\boldsymbol{K}_{\mathrm{i}}-\boldsymbol{K}_{\mathrm{f}}\right|$ is the nonrelativistic momentum transfer and the momentum vectors $\left(\boldsymbol{K}_{\mathrm{i}}, \boldsymbol{K}_{\mathrm{f}}\right)$ are related by the following formula:

$$
\boldsymbol{K}_{\mathrm{f}}=\left(\left|\boldsymbol{K}_{\mathrm{i}}\right|^{2}-3 / 4\right)^{1 / 2} .
$$

\section{Results and discussions}

In presenting our results it is convenient to consider separately those corresponding to nonrelativistic regime (the relativistic parameter $\gamma \approx 1$ ) and those related to relativistic one (the relativistic parameter $\gamma \approx 2$ ). Before beginning the discussion of the obtained results, it is worthwhile to recall the meaning of some abbreviation that will appear throughout this section. The NRDCS stands for the nonrelativistic differential cross-section, where nonrelativistic plane waves are used to describe the incident and scattered electrons. The SRDCS stands for the semirelativistic differential cross-section.

We begin our numerical work with the study of the dependence of the probability density for radial Darwin and Dirac wave functions on the atomic charge number $Z$. So long as the condition $Z \alpha \ll 1$ is verified, the use of Darwin wave function does not have any influence at all on the results at least in the first order of perturbation theory. So, the semirelativistic treatment when $Z$ increases may generate large errors but not in the case of this work. In this paper, we cannot have numerical instabilities since there are none. For the sake of illustration, we give in Fig. 1 the behavior of the probability density for radial Darwin wave functions as well as that of the exact relativistic Dirac wave functions for different values of $Z$. As you may see, even if it is not noticeable in Fig. 1, there are growing discrepancies for $Z=10$ and these become more pronounced when $Z=20$. The QED formulation shows that there are relativistic and spin effects at the relativistic domain and the nonrelativistic formulation is no longer valid.

In the relativistic regime, the semirelativistic differential cross-section results obtained for the $1 s \longrightarrow 2 s$ 


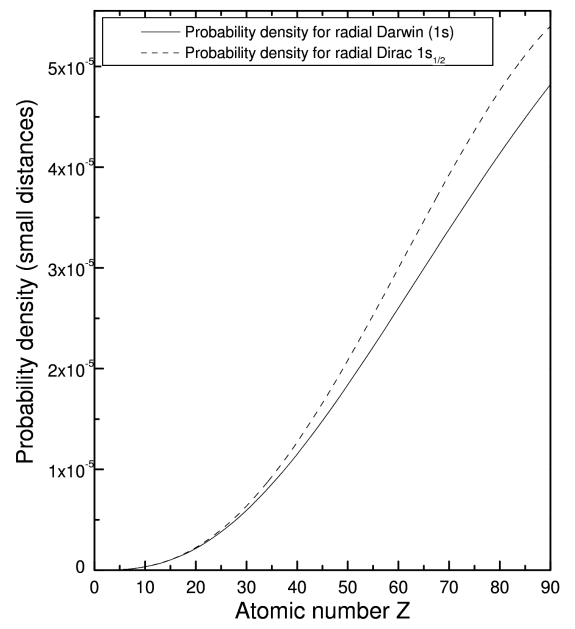

Fig. 1. Behavior of the probability density for radial Darwin wave function compared with that of the Dirac wave function for small distances and for increasing values of the atomic charge number.

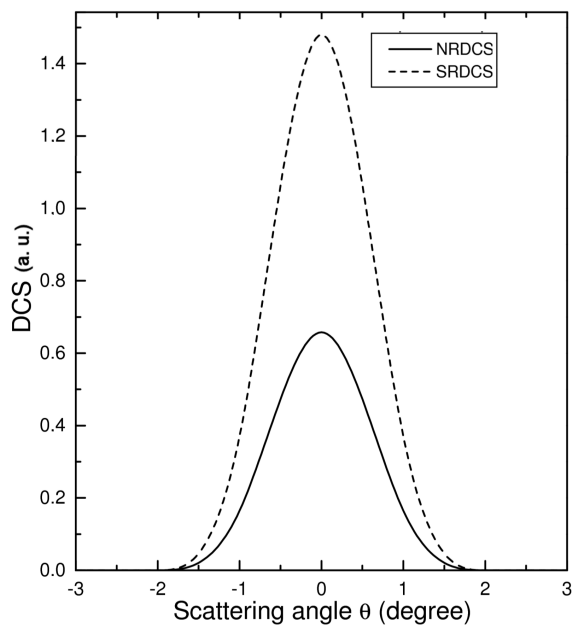

Fig. 2. The long-dashed line represents the semirelativistic DCS, the solid line represents the corresponding nonrelativistic DCS for a relativistic parameter $(\gamma=$ 1.5) as functions of the scattering angle $\theta$.

transition in atomic hydrogen by electron impact, are displayed in Figs. 2 and 3. In this regime, there are no theoretical models and experimental data for comparison as in nonrelativistic regime. In such a situation, it appears from Figs. 2 and 3 that in the limit of high electron kinetic energy, the effects of the additional spin terms and the relativity begin to be noticeable and that the nonrelativistic formalism is no longer applicable. Also a peak in the vicinity of $\theta_{\mathrm{f}}=0^{\circ}$ is clearly observed.

The investigation in the nonrelativistic regime was carried out with $\gamma$ as a relativistic parameter and $\theta$ as a scattering angle. In atomic units, the kinetic energy is related to $\gamma$ by the following relation: $E_{k}=c^{2}(\gamma-1)$. Figure 4 shows the dependence of DCS, obtained in two

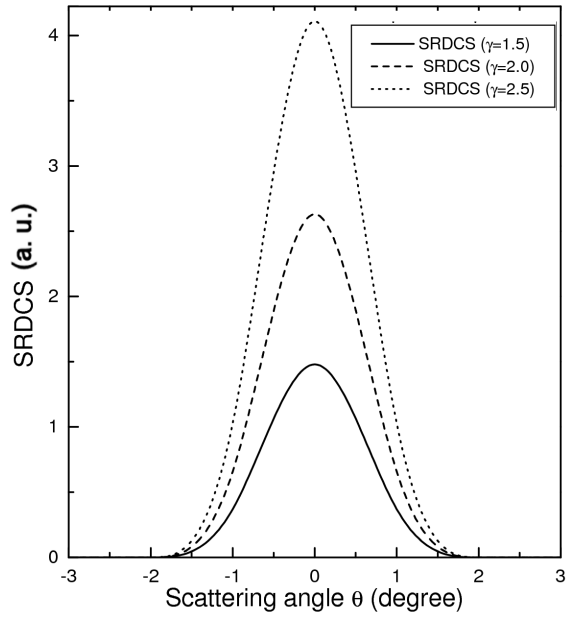

Fig. 3. The solid line represents the semirelativistic DCS, the long-dashed line represents the corresponding nonrelativistic DCS for various values of the relativistic parameter $(\gamma=1.5, \gamma=2$ and $\gamma=2.5)$ as functions of the scattering angle $\theta$.

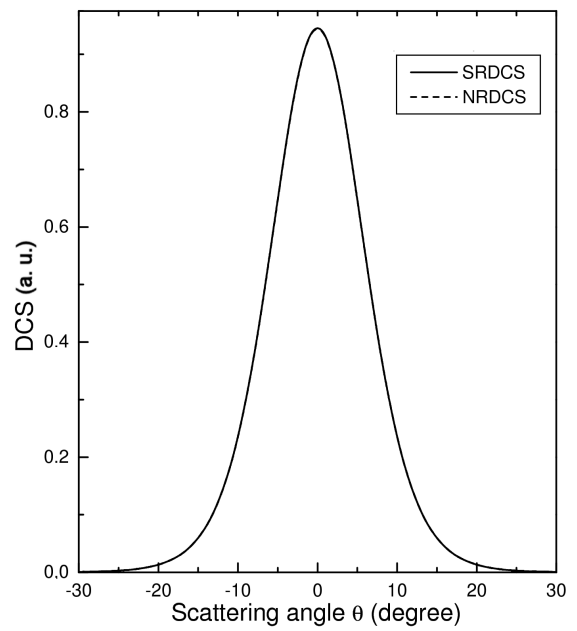

Fig. 4. The solid line represents the semirelativistic DCS, the long-dashed line represents the corresponding nonrelativistic DCS for a relativistic parameter $\gamma=$ 1.00053 as functions of the scattering angle $\theta$.

models (SRDCS, NRDCS), on scattering angle $\theta$. In this regime, it appears clearly that there is no difference between these models. Figure 5 shows the variation of the SRDCS with $\theta$ for various energies. It also shows that the SRDCS increases with $\gamma$, for small scattering angles and decreases elsewhere. Figure 6 presents the observed and calculated angular dependence of $1 s-2 s$ differential cross-section of $\mathrm{e}^{-}-\mathrm{H}$ scattering at incident energy $200 \mathrm{eV}$. Results obtained in the approaches (semirelativistic and nonrelativistic approximations) are indistinguishable and in good agreement with the experimental data provided by Williams [9]. 


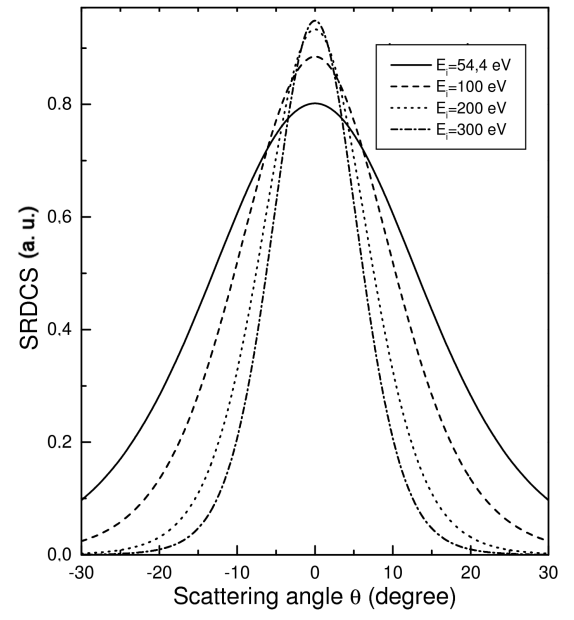

Fig. 5. The variation of the SRDCSs with respect to $\theta$, for various kinetic energies.

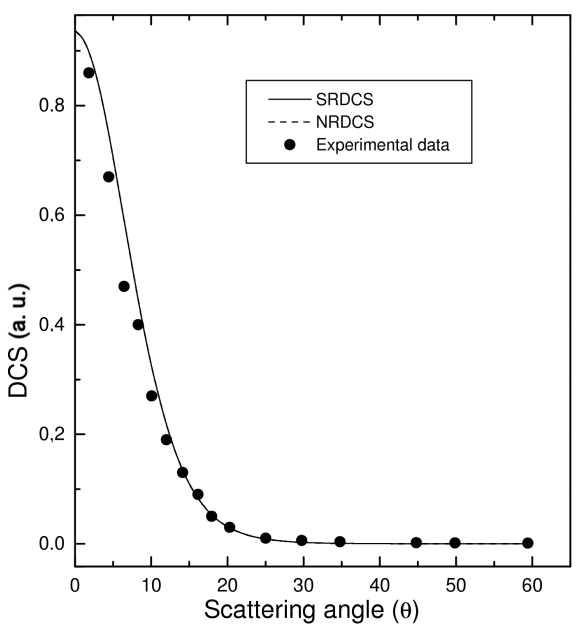

Fig. 6. The variation of the differential $1 s-2 s$ cross-section of $\mathrm{e}^{-}-\mathrm{H}$ scattering at $200 \mathrm{eV}$. The dots are the observed values of Williams [9]; the solid line represents the semirelativistic approximation and the long-dashed line corresponds to the nonrelativistic DCS.

\section{Conclusion}

In this paper we have presented the results of a semirelativistic excitation of atomic hydrogen by electronic impact. We have used the simple semirelativistic Darwin wave function that allows to obtain analytical results in an exact and closed form within the framework of the first Born approximation. This model gives good results if the condition $Z / c \ll 1$ is fulfilled. We have compared our results with previous nonrelativistic results and have found that the agreement between the different theoretical approaches is good in the nonrelativistic regime. We have also showed that the nonrelativistic treatment is no longer reliable for higher energies. We hope that we will be able to compare our theoretical results with forthcoming experimental data in the relativistic regime.

\section{Acknowledgments}

The authors would like to thank Prof. N. Bourima for his help in maintaining the text linguistically acceptable.

\section{References}

[1] R. Kisielius, K.A. Berrington, P.H. Norrington, J. Phys. B 28, 2459 (1995).

[2] N. Andersen, K. Bartschat, J. Phys. B 35, 4507 (2002).

[3] D. Payne, B. Krueger, K. Bartschat, J. Phys. B 38, 3349 (2005).

[4] Y. Attaourti, B. Manaut, A. Makhoute, Phys. Rev. A 69, 063407 (2004).

[5] J. Eichler, W.E. Meyerhof, Relativistic Atomic Collisions, Academic Press, San Diego 1995.

[6] F.W. Jr Byron, C.J. Joachain, Phys. Rep. 179, 211 (1989).

[7] L.S. Gradstein, I.M. Rizik, Tables of Integrals, Sutures, Sets and Their Products, Nauka, Moscow 1971.

[8] A.G. Grozin, Using Reduce in High Energy Physics, Cambridge University Press, Cambridge, England 1997.

[9] J.F. Williams, J. Phys. B 14, 1197 (1981). 